\title{
Origin of the Time Lag Phenomenon and the Global Signal in Resting-State fMRI
}

\author{
Shiori Amemiya*, Hidemasa Takao and Osamu Abe \\ Department of Radiology, Graduate School of Medicine, The University of Tokyo, Tokyo, Japan
}

The global mean signal of resting-state fMRI (rs-fMRl) shows a characteristic spatiotemporal pattern that is closely related to the pattern of vascular perfusion. Although being increasingly adopted in the mapping of the flow of neural activity, the mechanism that gives rise to the BOLD signal time lag remains controversial. In the present study, we compared the time lag of the global mean signal with those of the local network components obtained by applying temporal independent component analysis to the resting-state $\mathrm{fMRI}$ data, as well as by using simultaneous wide-field visual stimulation, and demonstrated that the time lag patterns are highly similar across all types of data. These results suggest that the time lag of the rs-fMRI signal reflects the

OPEN ACCESS

Edited by:

Peter Herman,

Yale University, United States

Reviewed by:

Blaise Frederick

Harvard Medical School,

United States

Yunjie Tong,

Purdue University, United States

*Correspondence:

Shiori Amemiya

amemiya-tky@umin.ac.jp

Specialty section:

This article was submitted to

Brain Imaging Methods,

a section of the journal

Frontiers in Neuroscience

Received: 18 August 2020 Accepted: 12 October 2020 Published: 29 October 2020

Citation:

Amemiya S, Takao $\mathrm{H}$ and Abe O (2020) Origin of the Time Lag Phenomenon and the Global Signal in Resting-State fMRI.

Front. Neurosci. 14:596084. doi: 10.3389/fnins.2020.596084 local variance of the hemodynamic responses rather than the arrival or transit time of the stimulus, whether the trigger is neuronal or non-neuronal in origin as long as it is mediated by local hemodynamic responses. Examinations of the internal carotid artery signal further confirmed that the arterial signal is tightly inversely coupled with the global mean signal in accordance with previous studies, presumably reflecting the blood flow or blood pressure changes that are occurring almost simultaneously in the internal carotid artery and the cerebral pial/capillary arteries, within the low-frequency component in human rs-fMRI.

Keywords: BOLD fMRI, autoregulation, neurovascular coupling, spatiotemporal dynamics, hemodynamics, global signal

\section{INTRODUCTION}

The advent of resting-state functional magnetic resonance imaging (rs-fMRI) has brought a means of investigating spontaneous neural activity and its macroscopic organization characterized by the coherence of the activity. The spatial patterns identified as areas with synchronous oscillation of the blood oxygenation level-dependent (BOLD) signal are termed resting-state networks (RSNs) (Fox et al., 2005). These networks are closely related to the anatomical connectivity among the neural subsystems that have been revealed by a wide variety of visual, sensorimotor, and cognitive task paradigms (Vincent et al., 2007; Zhang et al., 2010). However, the neurophysiology of the phenomenon, or the mechanism that controls and coordinates the intrinsic synchronization within and across neural systems, largely remains to be established (Drew et al., 2020). Undoubtedly, the successful mapping of the flow of information among the population of neurons would be the key to understanding all these and other enduring questions in neuroscience. Against this backdrop, attempts have been made to map the flow of neural signals by analyzing the spatiotemporal patterns 
of the rs-fMRI BOLD signal (Majeed et al., 2009, 2011; Mitra et al., 2014, 2015; Amemiya et al., 2016, 2019; Takeda et al., 2016; Belloy et al., 2018; Abbas et al., 2019; Raut et al., 2019).

Several animal studies have reported the existence of the global propagation of the intrinsic neural activities (Stroh et al., 2013; Matsui et al., 2016; Vanni et al., 2017) and that the time lag of the BOLD signal could correspond to such electrophysiological infra-slow activity (Matsui et al., 2016). However, such findings were not necessarily consistent with the time lag phenomenon in human fMRI studies. In the studies exploring the existence of the global signals of neural origin, rs-fMRI time lag structures were found to be multi-dimensional (Mitra et al., 2014, 2015; Amemiya et al., 2016). However, such global signals were not identified even when a temporal independent component analysis (ICA) was applied to allow detection of the overlapping activities (Amemiya et al., 2019) - contradicting the assumptions that there are multiple paths of the infra-slow neural activities independent of the underlying vascular dynamics.

BOLD fMRI is an indirect measurement of the neural activity exploiting a vascular response that is necessarily influenced by vascular dynamics. Accordingly, the validity of the inferences is crucially dependent on accurate estimates of the hemodynamic responses. The assumption that global mean signal (GMS) regression can eliminate the underlying vascular effect might lack adequate empirical support or conceptual plausibility. Therefore, not only from a physiological point of view, it is of practical importance in neuroimaging studies to understand the mechanism that gives rise to the global signals and their time lag, regardless of their origin.

Several lines of evidence to date indicate the contribution of physiological noise to the GMS (Liu T.T. et al., 2017). Interestingly, the GMS exhibits a constant time lag, as if it were traveling through the cerebrovascular system. In a series of studies on low-frequency oscillations of the BOLD signal, Tong et al. demonstrated that the signal measured in the peripheral vasculature using near-infrared spectroscopy is well correlated with the signal from the cerebrovascular system (Tong and Frederick, 2010, 2012, 2014a,b; Tong et al., 2013; Tong and Hocke, 2014). Furthermore, independent clinical studies measuring the rs-fMRI signal time lag using GMS as the reference signal have consistently identified rs-fMRI signal delay under hypoperfusion or ischemia comparable to that in dynamic susceptibility contrast (DSC) perfusion imaging (Lv et al., 2013; Amemiya et al., 2014; Christen et al., 2015). The delay was even evident in MR-defined ischemic penumbra or stroke core (Lv et al., 2013; Amemiya et al., 2014), where normal spontaneous neuronal activity is known to be absent, supporting the view that the major source of the GMS and its time lag are vascular in origin.

Under physiological conditions, the local vascular mean transit time is almost homogenous across the cerebral cortex and prolongs monotonically in proportion to the cerebral perfusion pressure decrease (Sette et al., 1989; Ito et al., 2003; Ibaraki et al., 2007). Consequently, the arrival and peak time of the venous flow are generally dependent on those of the arterial flow. That is, if the source of the signal simply flows through the vessels from the arteries to veins like a contrast agent, as in DSC perfusion imaging, the BOLD signal time lag would also reflect the arterial arrival and peak time difference, which is up to $500 \mathrm{~ms}$ (Hendrikse et al., 2008; MacIntosh et al., 2010), though the peak time difference would be amplified in the veins.

Alternatively, the GMS time lag might mainly reflect the variance in local hemodynamic responses. Major candidate sources of the GMS, such as the cardiac and respiratory factors, are known to cause local hemodynamic responses that are similar to neurovascular coupling, as is seen in the experimental manipulation of these factors (Battisti-Charbonney et al., 2011). For example, vasodilation in response to arterial partial pressure of carbon dioxide mainly occurs at the arterioles and precapillary sphincter (Ainslie and Duffin, 2009). Therefore, if it is the factor that triggers the GMS, in addition to the traveling time difference of the carbon dioxide, we need to take into account the local variance of the hemodynamic responses.

In this study we hypothesized that the BOLD time lag phenomenon is related to the variance of the local hemodynamic responses, which can vary on the order of seconds, rather than the time lag on the part of the stimulus or trigger, whether it is neuronal or non-neuronal in origin. Indeed, a similar pattern of the time lag has been shown in studies examining the BOLD signal changes associated with end-tidal carbon dioxide or with a breath-holding challenge (Chang and Glover, 2009). Although the time lag is less conspicuous, a similar contrast between the primary and association cortices has also been demonstrated in the temporal parameters of the hemodynamic response function (HRF) in task fMRI (Taylor et al., 2018). Therefore, it seems possible that the time lag phenomenon is caused by a substantially synchronous stimulus producing time lag depending on the local arterial density, or more precisely, the time required to increase the local blood flow, which is also dependent on the status of the arterioles of capillaries in terms of the vascular reactivity in each region (Roc et al., 2006; Bonakdarpour et al., 2007; Amemiya et al., 2012). Once the delay is embedded in its signal, the venous blood further drains into larger veins and sinuses carrying the BOLD signal as it is just like the contrast agent. The term substantial is used here to allow for the possibly traveling stimulus on the order of milliseconds, which is unlikely to be reflected in the time lag in this scenario. Another point to note is that BOLD signal time lag measurement is unlikely to distinguish the traveling time of a stimulus if carried out along with the blood flow before the induction of hemodynamic response time lags in the local veins because the two measures are likely proportionate to each other.

To test the hypothesis, we first examined the presence of the time lag phenomenon in the rs-fMRI local components obtained by applying the temporal ICA to the Human Connectome Project (HCP) rs-fMRI datasets (Amemiya et al., 2019). As previously well-explored in Smith et al. (2012), these components could subdivide and/or reorganize the conventional RSNs. Nevertheless, they are similar to the conventional RSNs in that they are confined within the functionally relevant cerebral structures as opposed to the GMS, which is almost ubiquitous in the systemic vasculatures (Tong and Frederick, 2010; Tong et al., 2012; Li et al., 2018). If the GMS time lag is the reflection of the "traveling signal," while the local network activities are synchronous and non-traveling as measured using magnetoencephalography (Brookes et al., 2011) 
or electroencephalography (Liu Q. et al., 2017), a similar time lag would not be found for the local components. In contrast, if the time lag is produced by the variance in the vascularity, resulting in the varied HRF, a similar time lag would also be found for the synchronized network signals.

The investigation of the resting-state local signal time lag might be more beneficial in terms of rs-fMRI methodology. However, the problem is that many independent components (ICs) are confined within areas with a similar global signal time lag, limiting the verification of our hypothesis. The overall ambiguity of the source of signals in rs-fMRI could be another problem. Therefore, in our second experiment, we also performed the task fMRI to further compare the time lag of the signals resulting from neural activation with that of the restingstate GMS. For this purpose, we prepared a projection system that enables the wide-field visual stimulation to activate the whole visual cortex simultaneously so that the findings would be more conclusive. Lastly, to address the question regarding the origin of the GMS, we examined the internal carotid artery signal using the rs-fMRI data acquired in Experiment 2. In addition to the time lag analysis following the procedure in previous studies (Tong et al., 2019b; Yao et al., 2019), we included some evaluation of the characteristics of the arterial signal to further clarify the origin of the phenomenon.

\section{MATERIALS AND METHODS}

\section{Experiment 1. Resting-State Functional Networks' Time Lag Mapping Data}

The data are originally from the WU-Minn HCP young healthy adults (ages 22-35) S1200 release that provides a paired dataset of the same group of subjects (day 1 and day 2). We used the same dataset previously analyzed with temporal ICA to examine the global and local signal contributions to the RSN synchronization (Amemiya et al., 2019). Briefly, the data of 50 subjects who underwent $3 \mathrm{~T}$ resting-state fMRI sessions without quality control issues, and whose mean framewise displacement was less than $0.2 \mathrm{~mm}$, were included in the analysis. All preprocessing and data analyses were performed for each dataset, respectively, in the same way.

\section{fMRI Data Analysis}

HCP imaging and pre-processing protocol have been previously described in detail (Glasser et al., 2013; Smith et al., 2013; Van Essen et al., 2013; Griffanti et al., 2014; Salimi-Khorshidi et al., 2014). Further pre-processing and analysis of the data were performed using tools from the AFNI libraries and inhouse scripts written and implemented in MATLAB 9.3 and 9.7 (MathWorks, Natick, MA, United States), as is described elsewhere (Amemiya et al., 2019). In short, linear trends were removed from the HCP data that had been processed with subject-level ICA noise reduction (sICA + FIX), and the data were band-pass filtered at $0.01-0.1 \mathrm{~Hz}$. The pre-processed data were temporally concatenated across runs to create a single $4 \mathrm{D}$ dataset of 120,000 time points $(1,200$ frames $\times 50$ subjects $\times 2$ phaseencoding directions) for test (Dataset 1) and re-test (Dataset
2) dataset, respectively. For temporal ICA, we employed a strategy adapted from Smith et al. (2012) to perform groupwise spatial ICA in advance of the final temporal ICA using FastICA (Hyvärinen, 1999). The temporal ICA decomposition was based on the Icasso algorithm (Himberg et al., 2004) that estimates the most appropriate decomposition yielding a set of reproducible IC clusters, which found 28 and 30 components for each dataset, respectively.

For all temporal ICs, the time-series of each run, once concatenated to be subjected to a temporal ICA, were deconcatenated so that the following analyses could be performed for each run separately. For each IC, the time lag map was computed as the relative time lag $t$ that gives the best positive fit between each voxel's time-series and the time-shifted $( \pm 5.8 \mathrm{~s}$ or \pm 8 TR) IC time-series using cross-correlation analysis (Supplementary Figure 1). All other types of time lag maps in Experiments 1 and 2 were similarly computed by using either the GMS or the task-induced BOLD signal as the reference signal. All data were up-sampled to a resolution of $0.18 \mathrm{~s}$ (1/4 TR) for the analysis (Tong et al., 2017, 2019b; Yao et al., 2019). The magnitude map of each IC was then computed as the Pearson's correlation coefficients between each voxel's time-series and the IC time-series that was shifted as much as $t$. Based on the spatial pattern, 21 and 20 ICs, respectively, were identified as local functional network ICs rather than global signal components (Amemiya et al., 2019). The GMS was computed as the average signal within a gray matter mask that was created by thresholding Montreal Neurological Institute (MNI) template at 30\% or larger probability of being gray matter.

\section{Time Lag Comparison}

To examine the hypothesis that a similar time lag phenomenon is found in the local ICs, the zero-centered (by linear detrending) relative time lag of each IC signal was compared with that of the GMS voxel-wisely for each subject's individual run. For visualization and superimposition of the time lag maps, each IC time lag was adjusted so that the mean of the IC time lag range equals that of the GMS for each subject's data. To minimize the bias in the time lag estimation for each signal, the time-series correlation threshold was set at the Pearson's correlation coefficient of $r>0.3$ (Tong et al., 2017, 2019b; Yao et al., 2019), which overall corresponded to the adjusted Z-score of $>3(p<0.0027)$ computed by the $\mathrm{xDF}$ approach with the "adaptive truncation" methods for the regularization of the autocorrelation function (Afyouni et al., 2019). All the ICs whose average magnitude map had voxels that consistently survived the time-series correlation threshold across 100 runs were included in the analysis. The correlation between the two time lag sets that was computed as Pearson's correlation coefficient for each run was Fisher's Z transformed and tested by using a two-tailed $t$-test over runs against the null hypothesis of no correlation.

\section{Experiment 2. Visual Task and Resting-State fMRI Participants}

Nine healthy subjects (four men and five women; $32.5 \pm 5.6$ years of age), who gave written informed consent, participated in the study. All were free of abnormal neurological history, taking 
no medication and with normal or corrected-to-normal visual acuity. None showed any abnormality of the brain on an MRI. All procedures complied with the Declaration of Helsinki, and the Institutional Review Board of the University of Tokyo approved the study.

\section{Visual Stimulus}

The visual stimulus was back-projected using a video projector and a mirror $(300 \mathrm{~mm} \times 300 \mathrm{~mm})$ onto a rear-screen $(300 \mathrm{~mm} \times 200 \mathrm{~mm})$ that was set $12 \mathrm{~cm}$ above the subject, subtending a horizontal and vertical visual angle of 138 and 118 degrees, respectively. The participants viewed the screen from inside the head coil through glasses with a power of +6.0 diopters (MediGlasses, Cambridge Research Systems, Kent, United Kingdom) to focus on the stimulus. The projection system was made entirely of plastic and prepared according to Greco et al. (2016). The stimulus was based on a black and white checkerboard pattern comprising 24 segments and 26 rings, with the pattern reversed polarity at $6 \mathrm{~Hz}$. The stimulus was presented in a block design comprising periods of $12 \mathrm{~s}$ of activation and $36 \mathrm{~s}$ of controls repeated for six cycles (task 1) following $11 \mathrm{~s}$ of prescan and $6 \mathrm{~s}$ of control condition. For task 2, the same stimulus was presented for $6 \mathrm{~s}$, followed by $24 \mathrm{~s}$ of control, and repeated for ten cycles. During the control phase, a gray screen with the luminance equal to the average of the black and white checks was presented. The subjects were told to gaze at a central red fixation dot throughout the runs. Immediately after each run, the subjects were asked to wave their hands and to report wakefulness during the scan subjectively.

\section{Scanning}

For each subject, 6 (or 10 in two subjects) runs of visual task fMRI (3 runs of task 1 and task 2, respectively), a single run of rsfMRI, and high spatial resolution T1-weighted (MPRAGE) and T2-weighted imaging were performed with a whole-body 3.0T MR unit (MAGNETOM Skyra, Siemens Healthcare, Erlangen, Germany) using a 32-channel phased-array head coil. For the fMRI scans, we used a multiband echo-planar imaging (EPI) sequence employing a simultaneous multi-slice imaging technique (Breuer et al., 2006; Moeller et al., 2010; Setsompop et al., 2012; Cauley et al., 2014) (Multi-Band EPI Package, Release 016a VE11C, Center for Magnetic Resonance Research, University of Minnesota $)^{1}$. The acquisition parameters were as follows: $\mathrm{TR}=750 \mathrm{~ms} ; \mathrm{TE}=32 \mathrm{~ms}$; flip angle $=62^{\circ}$; matrix size $=70 \times 70 ; \mathrm{FOV}=210 \mathrm{~mm} \times 210 \mathrm{~mm}$ with $100 \%$ phase sampling and no interpolation; $3 \mathrm{~mm}$-thick sections with no gap; 60 sections; 400/392/408 frames (resting/task1/task2 fMRI); multiband factor 4; iPAT factor 2; blipped controlled aliasing in parallel imaging results in higher acceleration (CAIPIRINHA) phase shift of FOV/4; and a leakage block technique (Cauley et al., 2014) was used for reconstruction. A total of 3 and 7 runs were excluded from the analysis for task 1 and task 2, respectively, because of non-compliance with the instructions.

\footnotetext{
${ }^{1}$ https://www.cmrr.umn.edu/multiband/
}

\section{Data Analysis \\ Preprocessing}

All task and rs-fMRI data were processed using SPM12 (Wellcome Department of Cognitive Neurology, London, United Kingdom) implemented in MATLAB 9.7 (MathWorks, Inc., Natick, MA, United States). Data were corrected for differences in acquisition time between slices, realigned to the first volume to account for movement artifacts, spatially aligned with anatomic T1-weighted images, and then normalized to the MNI space via the unified segmentation approach (Ashburner and Friston, 2005) using the T1-weighted images and spatially smoothed with a Gaussian kernel with $8 \mathrm{~mm}$ full-width at halfmaximum.

\section{Visual activation area}

For task fMRI data, statistical analysis was performed in two stages in a mixed-effects model. In the first-level analysis, the visual task condition was defined. The canonical HRF was convolved with a sequence of delta functions to form covariates for the general linear model. Six motion parameters were also included as nuisance regressors in the model, and the data were high-pass filtered at $1 / 128 \mathrm{~Hz}$. In the second-level analysis, statistical parametric maps of the task condition from each run were tested with a $t$-test to obtain group-level activation maps. Since the activation patterns of task 1 and task 2 were similar, group analysis was performed combining the two firstlevel analysis results (number of runs: $28+24=52$ ). A mask of activation areas was made by including the voxels showing significant positive activation at a voxel-wise familywise error rate corrected threshold of $p<0.001$ with a cluster size of $>10$ voxels.

\section{Time lag comparison within the visual areas}

For each run of task and rs-fMRI, the time lag map was computed as described in Experiment 1 by using the averaged time-series within the mask as the reference signal for the task fMRI data and the GMS for the rs-fMRI data, respectively. The correlation between the two time lag sets that was computed as Pearson's correlation coefficient for each run was Fisher's Z transformed and tested by using a two-tailed $t$-test over runs against the null hypothesis of no correlation.

\section{The internal carotid artery signal}

The time lag of the arterial signal was also measured in the extracranial internal carotid arteries following the procedure described in Tong et al. (2019b) and Yao et al. (2019). The regions of interest (ROIs) were semi-automatically obtained by setting an intensity threshold within the areas surrounding each carotid artery using MPRAGE T1-weighted images, in which large arteries show high signal intensity due to the time-of-flight inflow effect. In addition to the time lag analysis, we compared the arterial signal with the signal of the surrounding tissue to further clarify the origin of the signal change. For each arterial ROI, a peripheral ROI of the surrounding tissues was created by inflating the arterial ROI that was subsequently subtracted by the original ROI. All ROIs were visually inspected, and contaminated vessels were carefully removed. The arterial and peripheral signals were measured using the non-warped and non-smoothed data. The average signal of the arterial and peripheral ROIs was measured 
for each internal carotid artery and compared using a paired $t$-test. Cross-correlation analysis was performed as described above to measure the time lag between the arterial signal (right and left carotid arteries) and the GMS for each subject.

\section{RESULTS}

\section{Experiment 1. Time Lag Map of the Resting-State Functional Networks (HCP Data)}

Of the 21 and 20 resting-state functional network ICs identified as the local components by applying temporal ICA to HCP datasets (Amemiya et al., 2019), 10 and 11 ICs, respectively, that survived the time-series correlation threshold of Pearson's $r>0.3$ across the 100 runs were included in the analysis. One IC that was judged as being a global component based on the spatial pattern in the previous study (Amemiya et al., 2019) but whose time lag was not significantly correlated with that of the GMS was also included in the analysis (Dataset 1, C05). The magnitude and time lag maps of the 11 ICs, and those of the GMS, are shown in Figure 1 and Supplementary Figure 2, respectively. All IC time lags were significantly correlated with that of the GMS $(r=0.37 \pm 0.17, p<0.001$; re-test, $r=0.31 \pm 0.13$, $p<0.001$; Figure 2 and Supplementary Figure 3, 2D histogram of the pooled individual data). A larger time lag correlation was seen for the ICs with larger areas of high magnitude, such as the sensorimotor (C05 in Dataset 1) or the visual cortex (Figure 1, C14 in Dataset 1; Supplementary Figure 2, C16 and C21 in Dataset 2). A composite map, incorporating all functional network (local IC) time lag maps for each dataset shows a marked similarity between the time lag maps of the functional network signals and that of the GMS (Dataset $1, r=0.82$, $p<2.2 \times 10^{-308}$; Dataset 2, $r=0.86, p<2.2 \times 10^{-308}$ ) (Figure 1 and Supplementary Figure 2). A larger time lag between the IC and GMS time lag was more likely to be found at the periphery of each region (Supplementary Figure 4).

\section{Experiment 2. Visual Task and Resting-State fMRI \\ Time Lag Map of the Visual Task fMRI}

The wide-field visual stimulation system (Figure 3A) activated the whole visual cortex from the primary to the high-order areas, including the hMT/V5 near the ascending limb of the inferior temporal sulcus (Dumoulin et al., 2000) as well as the lateral geniculate bodies. The statistical map of t-values thresholded at familywise error-corrected $p<0.001$ is visualized in Figure 3B. The mean time-series of each task averaged within the mask across all runs are plotted with the standard deviation (SD) (Figure 3C). The average time lag maps obtained with the time-series as the reference signals are shown in Figure 3D. These time lag maps of the BOLD response to the simultaneous neural activation were not only similar to each other $(r=0.95$, $p<2.2 \times 10^{-308}$ ) but also highly correlated with that of the resting-state fMRI GMS (task $1, r=0.64, p<9.5 \times 10^{-19}$; task 2, $r=0.61, p<3.4 \times 10^{-13}$ ) (Figures 3D,E). The two time lag measures showed high correlation even at the subject level (task 1 vs. GMS, $r=0.64 \pm 0.11$, range $0.47-0.88$; task 2 vs. GMS, $r=0.64 \pm 0.14$, range 0.39-0.92). The time lag maps of the GMS obtained from the nine scans were similar to those of the HCP datasets ( $r=0.60$ and 0.61 for Dataset 1 and Dataset 2, respectively). Each voxel's signal time-series was highly correlated with the reference time-series, and the strength of the correlation was not significantly correlated with the absolute time lag (task 1 , $r=-0.08, p<0.10$; task 2, $r=-0.05, p<0.20)$.

The average frame-wise displacements of the task 1 (28 runs), task 2 (24 runs), and resting-state fMRI scans ( 9 runs) were $0.10 \pm 0.02,0.10 \pm 0.02$, and $0.09 \pm 0.02 \mathrm{~mm}$, respectively.

\section{Experimental Arterial Signal}

Iinternal carotid artery ROIs of a representative case are shown in Figure 4A. The original EPI images showed high signal intensity in the external carotid arteries or the vertebral arteries in all the subjects (Figure 4B). Although the internal carotid artery was less conspicuous, presumably due to the susceptibility artifact caused by the inhomogeneity of the local magnetic field near the pharynx or by the higher flow, the average signal in the internal carotid artery was, on the whole, significantly higher compared with the peripheral tissues (paired $t$-test, $p<0.0073$ ) (Figure 4C).

The cross-correlation analysis found that the GMS of the rsfMRI was negatively correlated $(r<-0.3)$ with the arterial signal in 15 of 18 carotid arteries $(r=-0.57 \pm 0.13$ ) (Figures 4D,E), with an average time lag of $-2.7 \pm 1.36 \mathrm{~s}$. In the other three carotid arteries, the maximum correlation ranged from -0.19 to 0.58 , with a delay of 2.1-5.4 s. All of these arteries showed a lower signal to the periphery. The cross-correlation plot combining the all subject data shows a negative peak at $4 \mathrm{~s}$ (Figure 4F).

\section{DISCUSSION}

By examining the time lag of the resting-state functional network signals, we demonstrated that their spatiotemporal pattern is similar to that of the GMS. Given that the functional network components are locally confined and nonglobally traveling signals, reflecting the synchronous activities as measured using magnetoencephalography (Brookes et al., 2011) or electroencephalography (Liu Q. et al., 2017), their time lag is likely to reflect the local variance of the hemodynamic responses. This view was further confirmed more directly in Experiment 2, in which we showed that simultaneous visual stimulation results in a similar time lag within the visual cortex. Since differences in the response timing of the visual neurons to varied types of stimulation are less than $100 \mathrm{~ms}$ across the mammalian visual system (Nowak and Bullier, 1997; Bair et al., 2002), neural factors are less likely to cause time lag on the order of seconds in the HRF. Such a view is also supported by empirical data showing that the HRF onset or peak time, known to vary up to several seconds (Taylor et al., 2018), does not differ so much depending on their "functional" anatomy (Puckett et al., 2014).

These results suggest that the time lag of the rs-fMRI signal reflects the local variance of the hemodynamic responses rather 


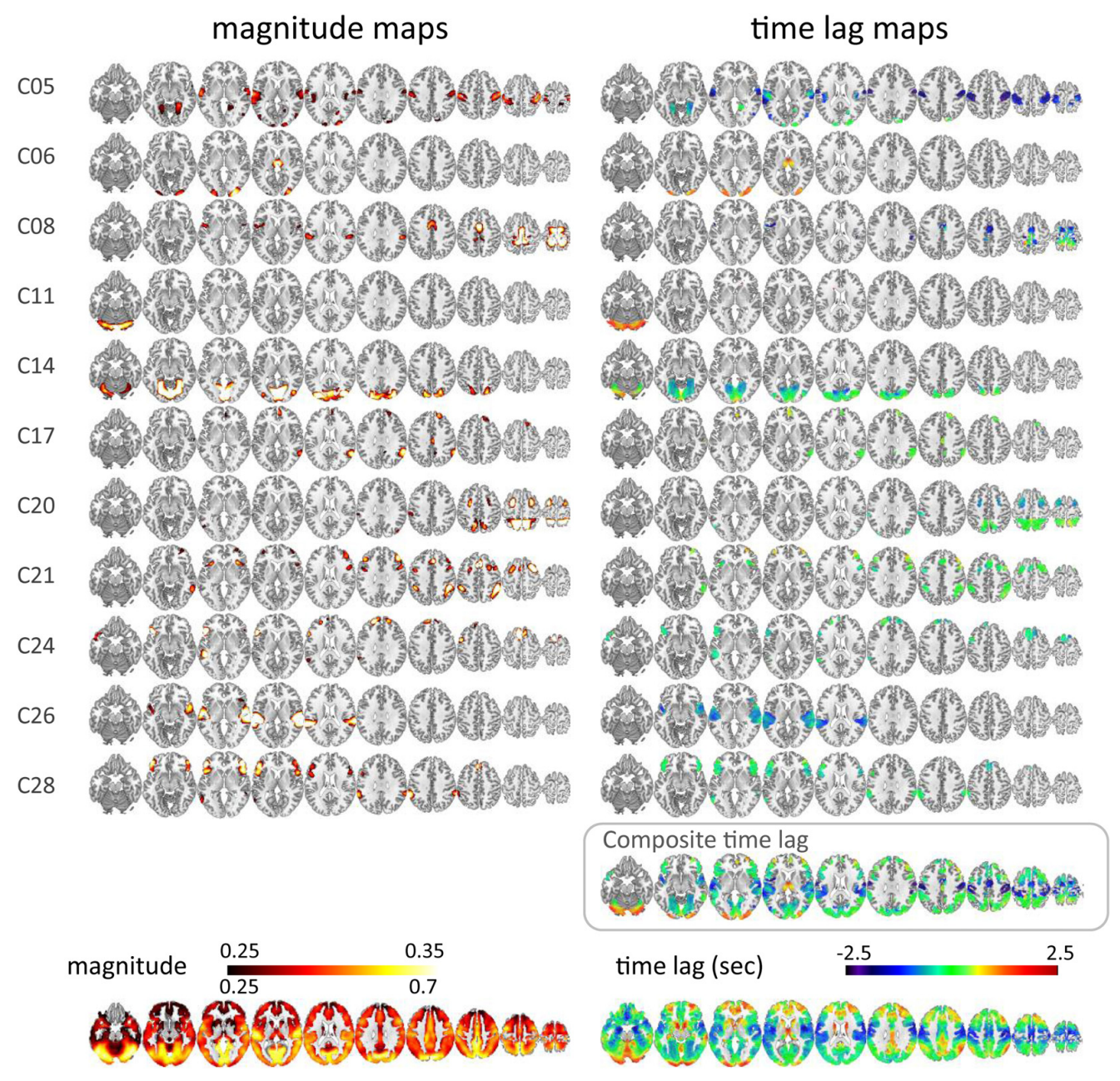

Global Mean Signal

FIGURE 1 | Time lag map of the resting-state functional networks (HCP data, Dataset 1). The magnitude (time-series correlation) and the time lag maps of the 10 ICs from Dataset 1, which was judged as a local component in the previous study (Amemiya et al., 2019) and that survived the time-series correlation threshold of Pearson's $r>0.3$ across the 100 runs are shown in the left and the right column, respectively. The bottom row is the magnitude and time lag maps of the global mean signal. A larger time lag correlation was seen for the ICs with larger areas of high magnitude, such as the sensorimotor cortex (C05) or the visual cortex (C14). $\mathrm{CO5}$ is the component originally judged as a global component whose time lag pattern was not significantly correlated with that of the global mean signal. The composite map incorporating all IC lag maps shows a marked similarity between the time lag maps of the local network signals and that of the global mean signal $\left(r=0.82, p<2.2 \times 10^{-308}\right)$.

than the arrival or transit times of the stimulus, which is in contrast to the general assumptions made in previous studies.

The local hemodynamic response to neural stimulation measured as BOLD signal change is sensitive to the vascularity in each region. It is delayed in the areas supplied by the small (collateral) arteries that are often found distal to the obstructed or narrowed arteries in ischemic patients (Roc et al., 2006; Bonakdarpour et al., 2007; Amemiya et al., 2012). In healthy subjects, the areas showing the BOLD signal delay seem to correspond well to the watershed areas. Located at the borders of the perfusion territories, these areas supplied by the most distal and smallest branches of the major arteries are known as the regions most vulnerable to a sudden drop in blood pressure or proximal occlusion of the major arteries (Wodarz, 1980; Zülch,
1985; Hossmann and Heiss, 2016). In the frontal lobes, the areas extending from the anterior horn of the lateral ventricle to the cortex showing delayed BOLD signal (Figure $\mathbf{1}$ and Supplementary Figure 2) correspond to the anterior watershed areas between the anterior and middle cerebral artery territories. On the other hand, the parieto-temporo-occipital wedge-shapeddelayed regions extending from the occipital horn of the lateral ventricle to the cortex (Figure 1 and Supplementary Figure 2) correspond to posterior watershed areas (Wodarz, 1980; Zülch, 1985; Hossmann and Heiss, 2016). Importantly, given that the BOLD response is dependent on the local hemodynamics, it is considered the local vascularity rather than the geographical localization of each region that matters. That is, the reason for the delayed BOLD signal in the association cortex seems not only 

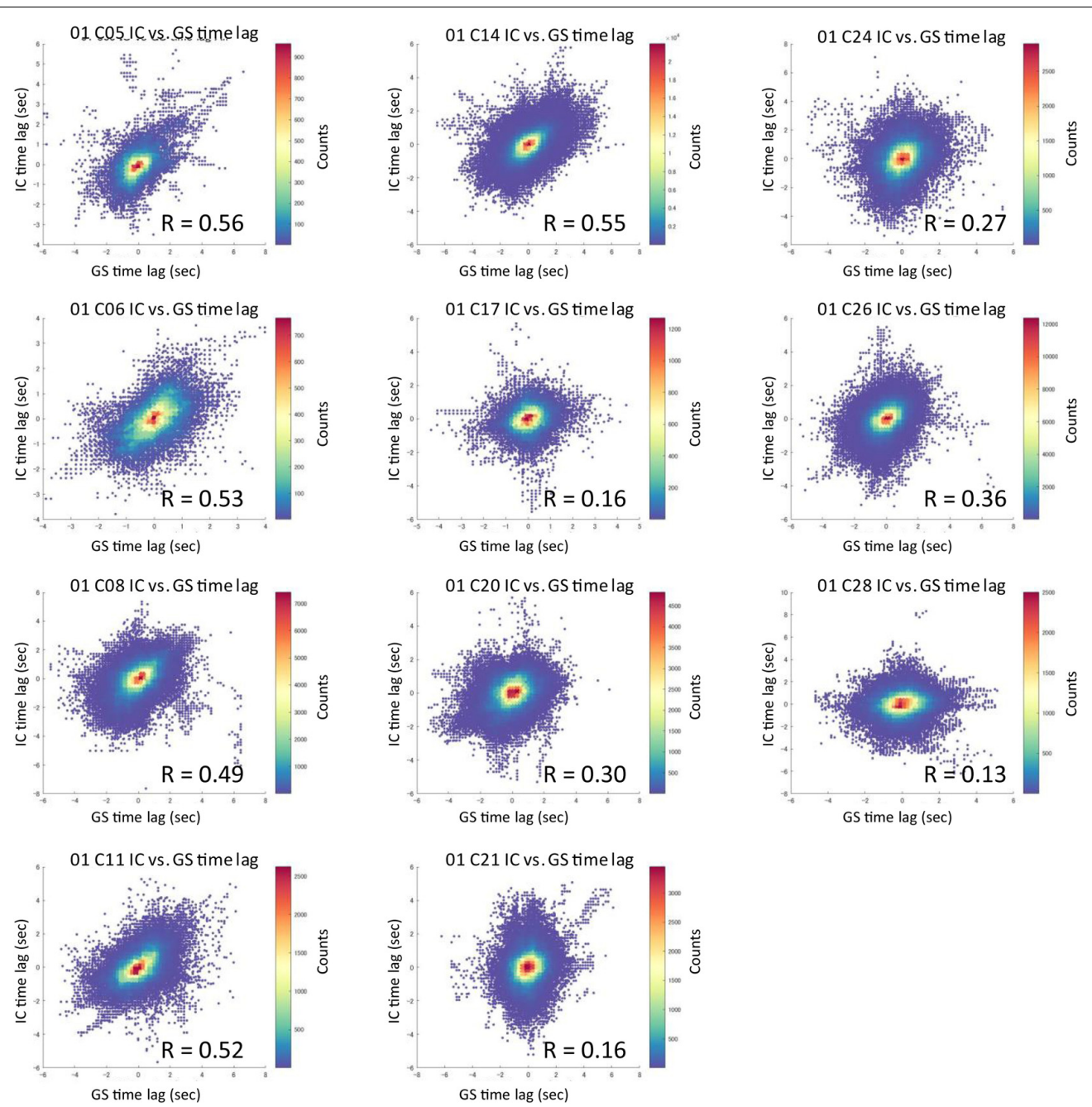

FIGURE 2 | 2D histogram of the pooled time lag data (HCP data, Dataset 1). For each IC, a 2D histogram shows the relationship between the IC vs. global mean signal time lag (pooled data across subjects). All IC time lags were significantly correlated with that of the global mean signal when compared within the areas surviving the time-series correlation threshold of Pearson's $r>0.3(r=0.37 \pm 0.17, p<0.001)$ (GMS = global mean signal).

because the arrival time of the blood from the heart is longer but also because the vascularity or the arteriolar/capillary density is lower in the higher-order regions compared with the primary cortices (Harrison et al., 2002; Weber et al., 2008).

These observations offer some insights into the origin of the GMS. Given that the source of the signal can be substantially synchronous across the cerebrovascular system or their traveling time lag is blood flow perfusion dependent, most cardiac and respiration related factors are the likely candidates of the phenomenon. In humans, oscillations in heart rate and blood pressure represent both autonomic neural fluctuations and mechanically induced central blood volume changes in synchrony with respiration (Cohen and Taylor, 2002). The interrelated factors, namely, the variations of the heart rate (Chang et al., 2009) and the blood pressure (Zhang et al., 2000), respiration volume per time (Birn et al., 2006, 2008) and partial pressure of end-tidal carbon dioxide (Wise et al., 2004) are indeed known to alter the cerebral blood flow or blood volume, thereby affecting the BOLD signal in the lowfrequency range of interest. Importantly, all these factors are considered to affect the cerebrovascular system substantially simultaneously (or with a perfusion-dependent traveling time lag in case of carbon dioxide). The abrupt change in blood pressure is associated with the immediate change in the blood flow, with the time lag between the arterial and the venous tracing of $0.2 \mathrm{~s}$ and with a regulatory reaction measured as the flow velocity beginning at 0.95 and $2.6 \mathrm{~s}$ for the middle cerebral artery and the straight sinus, respectively (Aaslid et al., 1991). The findings suggest that the vessels are stiff enough to immediately convey the pressure change to the periphery but that the changes in the flow associated with the hemodynamic response may take several seconds to be observed in the BOLD signal (note that the propagation of flow changes and transit time of the blood are different measures).

The physiological changes, even being synchronously induced in the capillary or pial arterioles just as in the case of the 
A

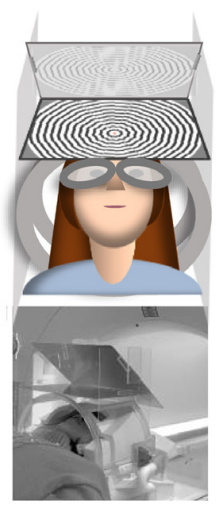

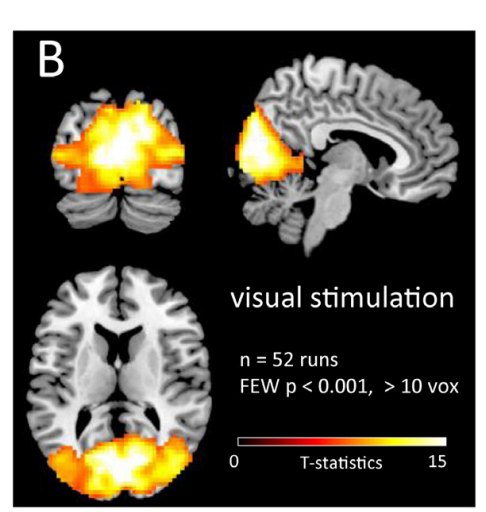

C

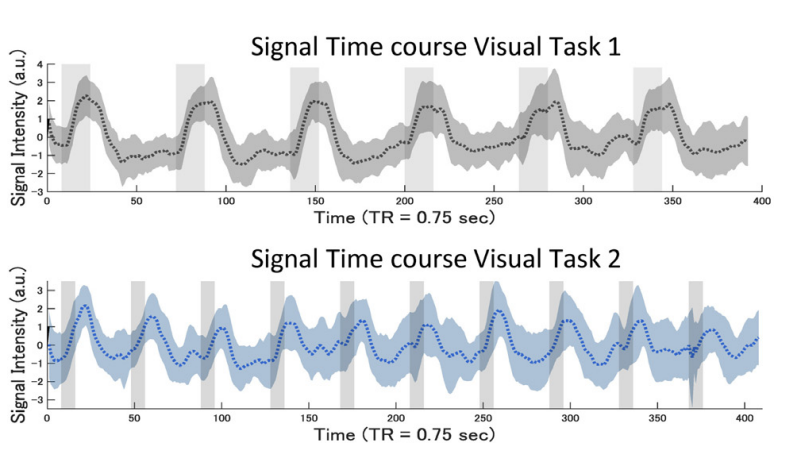

D

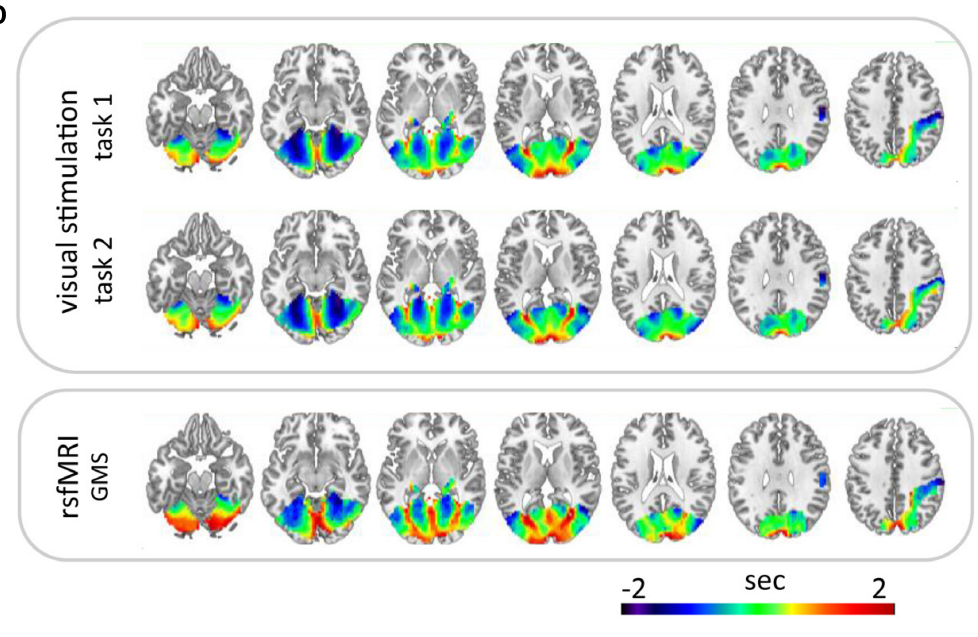

E
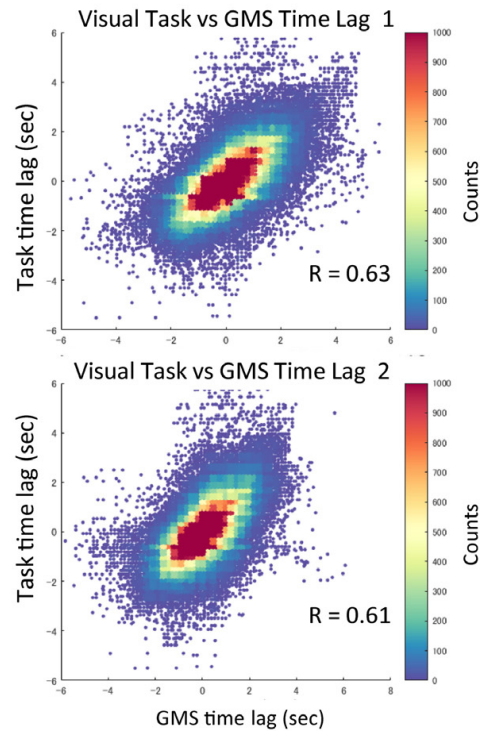

FIGURE 3 | Time lag of visual stimulation data. The wide-field visual stimulation system (A) activated the whole visual cortex from the primary to the high-order areas, including the hMTN5 as well as the lateral geniculate bodies [(B) t-map, familywise error-corrected $p<0.001]$. The mean time-series of each task averaged within the mask across all runs are plotted as a dotted line with SD indicated as shading (C). The average time lag maps obtained by using the mean time-series as the reference signals are shown in panel (D). These time lag maps of the BOLD response to the simultaneous neural activation were highly correlated with the time lag map of the resting-state global mean signal (task $1, r=0.64, p<9.5 \times 10^{-19}$; task $2, r=0.61, p<3.4 \times 10^{-13}$ ) [(E) $2 \mathrm{D}$ histogram of the pooled time lag data across subjects].

synchronous neural activation, would cause the local cerebral blood flow change, with a varied time lag depending on the arterial density in each region. The changes in the blood flow would be reflected, almost as they are, in the BOLD signal changes in the local veins given that the (spontaneous) BOLD signal is known to be significantly synchronized with the cerebral blood flow fluctuations in most cortical areas (Tak et al., 2014, 2015; Chen et al., 2015; Chiacchiaretta et al., 2018). Consistent with the hypothesis, direct observation of the low-frequency hemodynamic oscillations in mice using optical intrinsic signal imaging showed global fluctuations of the oxy-hemoglobin concentration (Bumstead et al., 2017), with a short time lag $(0.8 \mathrm{~s})$ between the central and peripheral regions of the anterior circulation territory (the medial regions seem to correspond the watershed of the middle and anterior cerebral artery's perfusion territory referring to the vascular anatomy in mice) (Xiong et al., 2017). Another awake human study investigating the $0.1 \mathrm{~Hz}$ hemodynamic oscillations using optical intrinsic signal imaging also showed a time lag of the signal in the frontal lobe (Rayshubskiy et al., 2014). Based on the article figures, the spatiotemporal pattern of the oscillations seems to share similar characteristics with the GMS in that the early signal is seen in the area closer to the primary motor cortex, which is found in the posterior part of the imaging field of view. However, given that the time lag in this pathological case (the entire field of view corresponds to the tumor area) seems to be much longer and that the imaging field was relatively limited, it might be difficult to conclude whether the pial arterial flow or diameter changes detected in the study correspond to the same time lag phenomenon.

While the physiological factors are also expected to affect the other arteries, the resultant changes are known to vary depending 


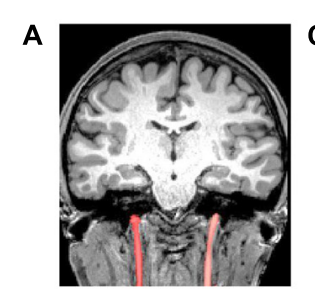

B

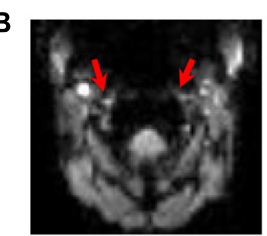

E

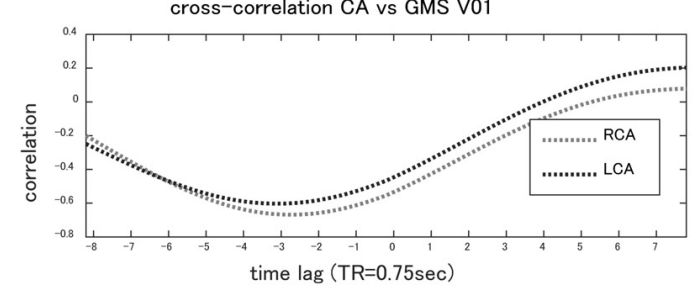

carotid artery \& peripheral signal V01

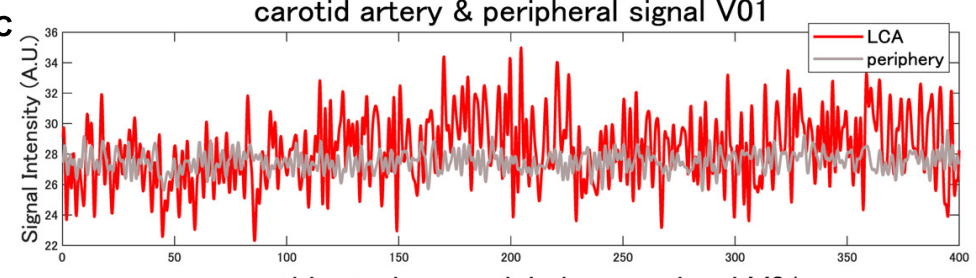

carotid arteries vs. global mean signal V01

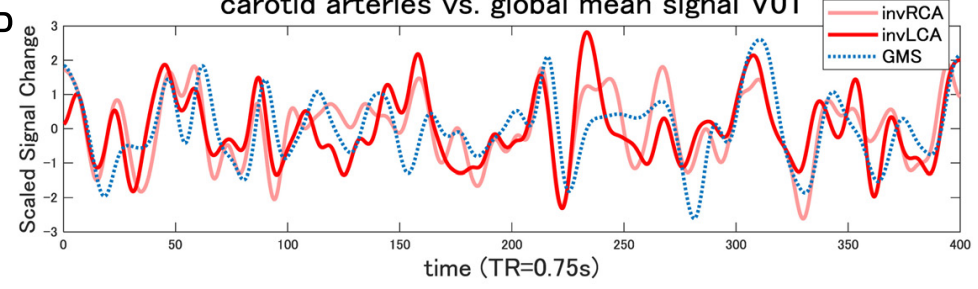

F

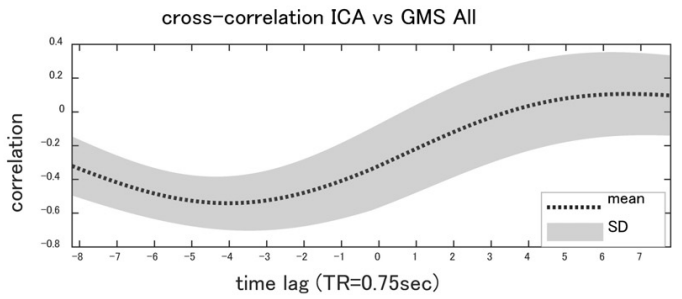

FIGURE 4 | Internal carotid artery signal. The coronal T1-weighted image (MPRAGE) shows the ROI of the bilateral internal carotid arteries (CAs) of a representative case (A). The original EPI image shows high signal intensity in the external carotid arteries or in the vertebral arteries (B). Although the internal carotid arteries (red arrows) were less conspicuous (B), the average signal within the internal carotid artery was higher compared with the peripheral tissues (C) in the majority of the cases (paired t-test, $p<0.0073$ ). Panel (D) shows the time-series of the global mean signal (GMS) and those of the internal carotid arteries of the same case. The cross-correlation analysis found that the global mean signal of the rs-fMRI was negatively correlated $(r<-0.3)$ with the arterial signal (E) in the majority of the cases, with an average time lag of $-2.7 \pm 1.36 \mathrm{~s}$. The average cross-correlation with the standard deviation (SD) is plotted in panel (F). The arterial signals are inversed in panel (D) to facilitate the comparison (invRCA and LCA = inversed right and left carotid artery).

on the type of the arteries. Previous studies on the autoregulation have revealed that the cerebral blood flow velocity, as measured using transcranial doppler ultrasonography, is modulated by the arterial blood pressure fluctuations in the major cerebral arteries (Aaslid et al., 1989, 1991; Tiecks et al., 1995; Kuo et al., 1998), especially at the frequency range of $0.07-0.3 \mathrm{~Hz}$ in humans (Zhang et al., 1998). On the other hand, the diameter of the middle cerebral artery is known to remain practically constant during different autoregulation tests in human studies (Huber and Handa, 1967; Aaslid et al., 1991; Giller et al., 1993; Tiecks et al., 1995) in contrast to the $5-10 \%$ change reported in studies using small animals whose middle cerebral artery diameter is less than $1 \mathrm{~mm}$ (Kontos, 1989) or to the spontaneous diameter changes of the pial arterioles up to $50 \%$ in awake mice (Drew et al., 2011). Blood flow response to carbon dioxide is also known to differ even among the internal, external, and vertebral carotid arteries in humans (Sato et al., 2012).

Such a difference in the form of response might explain why the internal carotid artery signal is highly inversely correlated with the GMS. In the internal carotid arteries, unlike smaller cerebral arteries, changes in the mean arterial pressure are known to be inversely correlated with the diameter, while positively correlated with the blood flow velocity, resulting in a stable flow (Liu et al., 2013). The signal within the carotid artery ROI is supposed to decrease if it sufficiently constricts
(Supplementary Figures 5A,B), given the present study result showing that the carotid artery demonstrates higher intensity compared with the surrounding tissues (Figure 4B). As for carbon dioxide-mediated changes, however, reports on the diameter of the internal carotid arteries are not consistent. While some showed that the internal carotid arteries dilate in response to the increase in the partial pressure of arterial carbon dioxide (Willie et al., 2012; Hoiland et al., 2016), the others found no significant change in the diameter (Sato et al., 2012; Coverdale et al., 2015). Therefore, if the carbon dioxide is the leading cause, the signal decrease in the internal carotid arteries is more likely to be caused by the intra-voxel inhomogeneity due to high flow (Supplementary Figures 5A,C). In either case, while the increase in the blood pressure or the blood flow can at least theoretically decrease the internal carotid artery signal, the simultaneous change can increase the local cerebral blood flow within a severalsecond delay. This will increase the BOLD signal due to the larger effect on the part of the deoxyhemoglobin decrease that increases the signal compared with the venous diameter increase that decreases the signal in the veins (Kim and Ogawa, 2012; Supplementary Figures 5D,E).

The finding that the macroscopic pattern of the BOLD signal time lag is similar for the rs-fMRI signal and the signal caused by the synchronous neural activation provides some implications for fMRI studies. Firstly, if the study aims to map the flow of 
neural activation, the global signal regression is not enough to eliminate the vascular effect even if it is applied, taking into account its time lag (Erdogan et al., 2016), or by using ICA to avoid introducing known biases associated with this controversial approach (Murphy et al., 2009). The resultant component still has the vascular time lag embedded in its signal and requires the HRF correction. Secondly, a simple computation of the GMS time lag can provide temporal information about the whole brain HRF. Although the precise HRF can vary even depending on the task duration (Logothetis et al., 2001; Martindale et al., 2005; Kennerley et al., 2012; Martin et al., 2013), such subject- and site-specific information would be useful to predict the BOLD response in each region for each subject to improve the sensitivity of fMRI (Handwerker et al., 2004). Finally, it is important to note that the time lag mapping of the rs-fMRI data could be flawed if not properly thresholded, as well-described in Tong et al. (2019a). The inclusion of the spuriously correlated voxels into the time lag mapping produces "another path of the traveling signal" masquerading as the flow of neural activation (as we have shown in Dataset 1, C05). Based on the highly coherent nature of the temporal properties of the BOLD response across the triggers shown in the present study, in addition to the previous study confirming a similar trend for the multiple global signals (Amemiya et al., 2019), such a phenomenon less likely to represent the flow of neural signals at least as the constant and independent components in the neural band of the human rsfMRI data.

There are some technical considerations for the present study. Firstly, some local IC time lag map showed a relatively low correlation with that of the GMS. It might be due to a larger variance caused by the small size of the involved area. The high overall similarity between the composite maps of the local ICs and the GMS supports this view. It is also important to note that the correlation might be underestimated because we used HCP data pre-processed with sICA + FIX denoising. While the denoising strategy is beneficial in reducing motionrelated artifacts, it also removes signals form the large veins and venous sinuses. Although we measured the GMS within the gray matter, given that it is generally highly correlated with the venous signals (Tong et al., 2019a), some fraction of the GMS signal must have been removed thorough the process. Secondly, while the temporal ICA is suitable in finding spatially overlapping activities, it might more likely divide one component into multiple ICs due to a time lag. However, even if it were the case, it does not affect the comparison of the time lag structures. The same also holds if the assumed origin of the local ICs were not entirely neural. Finally, although the primary source of the GMS is likely the physiological noise, it could also have some neural components. Both electroencephalographic work in humans and microelectrode recordings in anesthetized monkeys have shown widespread BOLD fluctuations to be correlated with slow changes in neural activity (Leopold et al., 2003; He et al., 2008; Scholvinck et al., 2010; Liu et al., 2018).

The neurovascular coupling is a complex phenomenon, and a comprehensive picture of the cellular and vascular mechanisms is yet to be identified (Hillman, 2014; Drew, 2019). Although our view, based on the similarity of the BOLD responses, provides some insights into the origin of the global signal and the time lag phenomenon, it would be more informative if we will be able to also address the possible difference based on the mechanism triggering the vascular response using rs-fMRI.

\section{CONCLUSION}

By comparing the spatiotemporal characteristics of the rs-fMRI signal with those of the simultaneously induced neural signals, we have demonstrated that the time lag phenomenon in the rs-fMRI most likely reflects the regional variance in the hemodynamic responses rather than the time lag on the part of the stimulus or trigger and that the cause of the GMS can be substantially synchronous. The tight inverse coupling between the internal carotid artery signal and the GMS is presumably reflecting the blood flow or blood pressure changes that are occurring almost simultaneously in the internal carotid artery and the pial/capillary arteries within the $0.01-0.1 \mathrm{~Hz}$ low-frequency component in human rs-fMRI.

\section{DATA AVAILABILITY STATEMENT}

The raw data supporting the conclusions of this article will be made available by the authors, without undue reservation.

\section{ETHICS STATEMENT}

The studies involving human participants were reviewed and approved by University of Tokyo. The patients/participants provided their written informed consent to participate in this study.

\section{AUTHOR CONTRIBUTIONS}

SA contributed to the conception and design of the study, acquired data, performed the statistical analysis, and wrote the first draft of the manuscript. All authors contributed to the final drafting of the manuscript, read and approved the submitted version.

\section{FUNDING}

This work was supported by JSPS Grant-in-Aid for Scientific Research (C 18K07707) and grants from the Takeda Science Foundation, the Tokyo Society of Medical Sciences, and the Japanese Society of Neuroradiology to SA.

\section{SUPPLEMENTARY MATERIAL}

The Supplementary Material for this article can be found online at: https://www.frontiersin.org/articles/10.3389/fnins.2020. 596084/full\#supplementary-material 


\section{REFERENCES}

Aaslid, R., Lindegaard, K. F., Sorteberg, W., and Nornes, H. (1989). Cerebral autoregulation dynamics in humans. Stroke 20, 45-52. doi: 10.1161/01.str.20. 1.45

Aaslid, R., Newell, D. W., Stooss, R., Sorteberg, W., and Lindegaard, K. F. (1991). Assessment of cerebral autoregulation dynamics from simultaneous arterial and venous transcranial Doppler recordings in humans. Stroke 22, 1148-1154. doi: 10.1161/01.str.22.9.1148

Abbas, A., Belloy, M., Kashyap, A., Billings, J., Nezafati, M., Schumacher, E. H., et al. (2019). Quasi-periodic patterns contribute to functional connectivity in the brain. Neuroimage 191, 193-204. doi: 10.1016/j.neuroimage.2019.01.076

Afyouni, S., Smith, S. M., and Nichols, T. E. (2019). Effective degrees of freedom of the Pearson's correlation coefficient under autocorrelation. Neuroimage 199, 609-625. doi: 10.1016/j.neuroimage.2019.05.011

Ainslie, P. N., and Duffin, J. (2009). Integration of cerebrovascular $\mathrm{CO}_{2}$ reactivity and chemoreflex control of breathing: mechanisms of regulation, measurement, and interpretation. Am. J. Physiol. Regul. Integr. Comp. Physiol. 296, R14731495. doi: 10.1152/ajpregu.91008.2008

Amemiya, S., Kunimatsu, A., Saito, N., and Ohtomo, K. (2012). Impaired hemodynamic response in the ischemic brain assessed with BOLD fMRI. Neuroimage 61, 579-590. doi: 10.1016/j.neuroimage.2012.04.001

Amemiya, S., Kunimatsu, A., Saito, N., and Ohtomo, K. (2014). Cerebral hemodynamic impairment: assessment with resting-state functional MR imaging. Radiology 270, 548-555.

Amemiya, S., Takao, H., and Abe, O. (2019). Global vs. Network-specific regulations as the source of intrinsic coactivations in resting-state networks. Front. Syst. Neurosci. 13:65. doi: 10.3389/fnsys.2019.00065

Amemiya, S., Takao, H., Hanaoka, S., and Ohtomo, K. (2016). Global and structured waves of rs-fMRI signal identified as putative propagation of spontaneous neural activity. Neuroimage 133, 331-340. doi: 10.1016/j. neuroimage.2016.03.033

Ashburner, J., and Friston, K. J. (2005). Unified segmentation. Neuroimage 26, 839-851. doi: 10.1016/j.neuroimage.2005.02.018

Bair, W., Cavanaugh, J. R., Smith, M. A., and Movshon, J. A. (2002). The timing of response onset and offset in macaque visual neurons. J. Neurosci. 22, 3189-3205. doi: 10.1523/jneurosci.22-08-03189.2002

Battisti-Charbonney, A., Fisher, J., and Duffin, J. (2011). The cerebrovascular response to carbon dioxide in humans. J. Physiol. 589, 3039-3048.

Belloy, M. E., Naeyaert, M., Abbas, A., Shah, D., Vanreusel, V., van Audekerke, J., et al. (2018). Dynamic resting state fMRI analysis in mice reveals a set of Quasi-Periodic Patterns and illustrates their relationship with the global signal. Neuroimage 180(Pt B), 463-484. doi: 10.1016/j.neuroimage.2018.01.075

Birn, R. M., Diamond, J. B., Smith, M. A., and Bandettini, P. A. (2006). Separating respiratory-variation-related fluctuations from neuronal-activityrelated fluctuations in fMRI. Neuroimage 31, 1536-1548. doi: 10.1016/j. neuroimage.2006.02.048

Birn, R. M., Smith, M. A., Jones, T. B., and Bandettini, P. A. (2008). The respiration response function: the temporal dynamics of fMRI signal fluctuations related to changes in respiration. Neuroimage 40, 644-654. doi: 10.1016/j.neuroimage. 2007.11.059

Bonakdarpour, B., Parrish, T. B., and Thompson, C. K. (2007). Hemodynamic response function in patients with stroke-induced aphasia: implications for fMRI data analysis. Neuroimage 36, 322-331.

Breuer, F. A., Blaimer, M., Mueller, M. F., Seiberlich, N., Heidemann, R. M., Griswold, M. A., et al. (2006). Controlled aliasing in volumetric parallel imaging (2D CAIPIRINHA). Magn. Reson. Med. 55, 549-556. doi: 10.1002/mrm.20787

Brookes, M. J., Woolrich, M., Luckhoo, H., Price, D., Hale, J. R., Stephenson, M. C., et al. (2011). Investigating the electrophysiological basis of resting state networks using magnetoencephalography. Proc. Natl. Acad. Sci. U.S.A. 108, 16783-16788. doi: 10.1073/pnas.1112685108

Bumstead, J. R., Bauer, A. Q., Wright, P. W., and Culver, J. P. (2017). Cerebral functional connectivity and Mayer waves in mice: phenomena and separability. J. Cereb. Blood Flow Metab. 37, 471-484. doi: 10.1177/0271678x16629977

Cauley, S. F., Polimeni, J. R., Bhat, H., Wald, L. L., and Setsompop, K. (2014). Interslice leakage artifact reduction technique for simultaneous multislice acquisitions. Magn. Reson. Med. 72, 93-102. doi: 10.1002/mrm.24898
Chang, C., Cunningham, J. P., and Glover, G. H. (2009). Influence of heart rate on the BOLD signal: the cardiac response function. Neuroimage 44, 857-869. doi: 10.1016/j.neuroimage.2008.09.029

Chang, C., and Glover, G. H. (2009). Relationship between respiration, end-tidal CO2, and BOLD signals in resting-state fMRI. Neuroimage 47, 1381-1393. doi: 10.1016/j.neuroimage.2009.04.048

Chen, J. J., Jann, K., and Wang, D. J. (2015). Characterizing resting-state brain function using arterial spin labeling. Brain Connect. 5, 527-542. doi: 10.1089/ brain.2015.0344

Chiacchiaretta, P., Cerritelli, F., Bubbico, G., Perrucci, M. G., and Ferretti, A. (2018). Reduced dynamic coupling between spontaneous BOLD-CBF fluctuations in older adults: a dual-echo pCASL study. Front. Aging Neurosci. 10:115. doi: 10.3389/fnagi.2018.00115

Christen, T., Jahanian, H., Ni, W. W., Qiu, D., Moseley, M. E., and Zaharchuk, G. (2015). Noncontrast mapping of arterial delay and functional connectivity using resting-state functional MRI: a study in Moyamoya patients. J. Magn. Reson. Imaging 41, 424-430.

Cohen, M. A., and Taylor, J. A. (2002). Short-term cardiovascular oscillations in man: measuring and modelling the physiologies. J. Physiol. 542(Pt 3), 669-683. doi: 10.1113/jphysiol.2002.017483

Coverdale, N. S., Lalande, S., Perrotta, A., and Shoemaker, J. K. (2015). Heterogeneous patterns of vasoreactivity in the middle cerebral and internal carotid arteries. Am. J. Physiol. Heart Circ. Physiol. 308, H1030-H1038. doi: 10.1152/ajpheart.00761.2014

Drew, P. J. (2019). Vascular and neural basis of the BOLD signal. Curr. Opin. Neurobiol. 58, 61-69. doi: 10.1016/j.conb.2019.06.004

Drew, P. J., Mateo, C., Turner, K. L., Yu, X., and Kleinfeld, D. (2020). Ultraslow oscillations in fMRI and resting-state connectivity: neuronal and vascular contributions and technical confounds. Neuron 107, 782-804. doi: 10.1016/j. neuron.2020.07.020

Drew, P. J., Shih, A. Y., and Kleinfeld, D. (2011). Fluctuating and sensory-induced vasodynamics in rodent cortex extend arteriole capacity. Proc. Natl. Acad. Sci. U.S.A. 108, 8473-8478. doi: 10.1073/pnas.1100428108

Dumoulin, S. O., Bittar, R. G., Kabani, N. J., Baker, C. L. Jr., Le Goualher, G., Bruce Pike, G., et al. (2000). A new anatomical landmark for reliable identification of human area V5/MT: a quantitative analysis of sulcal patterning. Cereb. Cortex 10, 454-463. doi: 10.1093/cercor/10.5.454

Erdogan, S. B., Tong, Y., Hocke, L. M., Lindsey, K. P., and deB Frederick, B. (2016). Correcting for blood arrival time in global mean regression enhances functional connectivity analysis of resting state fMRI-BOLD signals. Front. Hum. Neurosci. 10:311. doi: 10.3389/fnhum.2016.00311

Fox, M. D., Snyder, A. Z., Vincent, J. L., Corbetta, M., Van Essen, D. C., and Raichle, M. E. (2005). The human brain is intrinsically organized into dynamic, anticorrelated functional networks. Proc. Natl. Acad. Sci. U.S.A. 102, 96739678.

Giller, C. A., Bowman, G., Dyer, H., Mootz, L., and Krippner, W. (1993). Cerebral arterial diameters during changes in blood pressure and carbon dioxide during craniotomy. Neurosurgery 32, 737-741; discussion 741-742.

Glasser, M. F., Sotiropoulos, S. N., Wilson, J. A., Coalson, T. S., Fischl, B., Andersson, J. L., et al. (2013). The minimal preprocessing pipelines for the human connectome project. Neuroimage 80, 105-124. doi: 10.1016/j. neuroimage.2013.04.127

Greco, V., Frijia, F., Mikellidou, K., Montanaro, D., Farini, A., D’Uva, M., et al. (2016). A low-cost and versatile system for projecting wide-field visual stimuli within fMRI scanners. Behav. Res. Methods 48, 614-620.

Griffanti, L., Salimi-Khorshidi, G., Beckmann, C. F., Auerbach, E. J., Douaud, G., Sexton, C. E., et al. (2014). ICA-based artefact removal and accelerated fMRI acquisition for improved resting state network imaging. Neuroimage 95, 232-247. doi: 10.1016/j.neuroimage.2014.03.034

Handwerker, D. A., Ollinger, J. M., and D’Esposito, M. (2004). Variation of BOLD hemodynamic responses across subjects and brain regions and their effects on statistical analyses. Neuroimage 21, 1639-1651. doi: 10.1016/j.neuroimage.2003. 11.029

Harrison, R. V., Harel, N., Panesar, J., and Mount, R. J. (2002). Blood capillary distribution correlates with hemodynamic-based functional imaging in cerebral cortex. Cereb. Cortex 12, 225-233. doi: 10.1093/cercor/ 12.3.225 
He, B. J., Snyder, A. Z., Zempel, J. M., Smyth, M. D., and Raichle, M. E. (2008). Electrophysiological correlates of the brain's intrinsic large-scale functional architecture. Proc. Natl. Acad. Sci. U.S.A. 105, 16039-16044.

Hendrikse, J., Petersen, E. T., Van Laar, P. J., and Golay, X. (2008). Cerebral border zones between distal end branches of intracranial arteries: MR imaging. Radiology 246, 572-580.

Hillman, E. M. (2014). Coupling mechanism and significance of the BOLD signal: a status report. Annu. Rev. Neurosci. 37, 161-181.

Himberg, J., Hyvarinen, A., and Esposito, F. (2004). Validating the independent components of neuroimaging time series via clustering and visualization. Neuroimage 22, 1214-1222. doi: 10.1016/j.neuroimage.2004.03.027

Hoiland, R. L., Tymko, M. M., Bain, A. R., Wildfong, K. W., Monteleone, B., and Ainslie, P. N. (2016). Carbon dioxide-mediated vasomotion of extracranial cerebral arteries in humans: a role for prostaglandins? J. Physiol. 594, 3463-3481. doi: 10.1113/jp272012

Hossmann, K. A., and Heiss, W. D. (2016). History of the Letzte Wiese/last meadow concept of brain ischemia. Stroke 47, e46-e50. doi: 10.1161/strokeaha.115. 010976

Huber, P., and Handa, J. (1967). Effect of contrast material, hypercapnia, hyperventilation, hypertonic glucose and papaverine on the diameter of the cerebral arteries. Angiographic determination in man. Invest. Radiol. 2, 17-32.

Hyvärinen, A. (1999). Fast and robust fixed-point algorithms for independent component analysis. IEEE Trans. Neural Netw. 10, 626-634.

Ibaraki, M., Ito, H., Shimosegawa, E., Toyoshima, H., Ishigame, K., Takahashi, K., et al. (2007). Cerebral vascular mean transit time in healthy humans: a comparative study with PET and dynamic susceptibility contrast-enhanced MRI. J. Cereb. Blood Flow Metab. 27, 404-413. doi: 10.1038/sj.jcbfm.9600337

Ito, H., Kanno, I., Takahashi, K., Ibaraki, M., and Miura, S. (2003). Regional distribution of human cerebral vascular mean transit time measured by positron emission tomography. Neuroimage 19, 1163-1169.

Kennerley, A. J., Harris, S., Bruyns-Haylett, M., Boorman, L., Zheng, Y., Jones, M., et al. (2012). Early and late stimulus-evoked cortical hemodynamic responses provide insight into the neurogenic nature of neurovascular coupling. J. Cereb. Blood Flow Metab. 32, 468-480. doi: 10.1038/jcbfm.2011.163

Kim, S. G., and Ogawa, S. (2012). Biophysical and physiological origins of blood oxygenation level-dependent fMRI signals. J. Cereb. Blood Flow Metab. 32, 1188-1206. doi: 10.1038/jcbfm.2012.23

Kontos, H. A. (1989). Validity of cerebral arterial blood flow calculations from velocity measurements. Stroke 20, 1-3. doi: 10.1161/01.str.20.1.1

Kuo, T. B., Chern, C. M., Sheng, W. Y., Wong, W. J., and Hu, H. H. (1998). Frequency domain analysis of cerebral blood flow velocity and its correlation with arterial blood pressure. J. Cereb. Blood Flow Metab. 18, 311-318.

Leopold, D. A., Murayama, Y., and Logothetis, N. K. (2003). Very slow activity fluctuations in monkey visual cortex: implications for functional brain imaging. Cereb. Cortex 13, 422-433.

Li, Y., Zhang, H., Yu, M., Yu, W., Frederick, B. D., and Tong, Y. (2018). Systemic low-frequency oscillations observed in the periphery of healthy human subjects. J. Biomed. Opt. 23, 1-11. doi: 10.1117/1.jbo.23.5.057001

Liu, J., Zhu, Y. S., Hill, C., Armstrong, K., Tarumi, T., Hodics, T., et al. (2013). Cerebral autoregulation of blood velocity and volumetric flow during steadystate changes in arterial pressure. Hypertension 62, 973-979. doi: 10.1161/ hypertensionaha.113.01867

Liu, Q., Farahibozorg, S., Porcaro, C., Wenderoth, N., and Mantini, D. (2017). Detecting large-scale networks in the human brain using high-density electroencephalography. Hum. Brain Mapp. 38, 4631-4643. doi: 10.1002/hbm. 23688

Liu, T. T., Nalci, A., and Falahpour, M. (2017). The global signal in fMRI: nuisance or information? Neuroimage 150, 213-229. doi: 10.1016/j.neuroimage.2017.02. 036

Liu, X., De Zwart, J. A., Schölvinck, M. L., Chang, C., Frank, Q. Y., Leopold, D. A., et al. (2018). Subcortical evidence for a contribution of arousal to fMRI studies of brain activity. Nat. Commun. 9:395.

Logothetis, N. K., Pauls, J., Augath, M., Trinath, T., and Oeltermann, A. (2001). Neurophysiological investigation of the basis of the fMRI signal. Nature 412, 150-157. doi: 10.1038/35084005

Lv, Y., Margulies, D. S., Cameron Craddock, R., Long, X., Winter, B., Gierhake, D., et al. (2013). Identifying the perfusion deficit in acute stroke with resting-state functional magnetic resonance imaging. Ann. Neurol. 73, $136-140$.

MacIntosh, B. J., Filippini, N., Chappell, M. A., Woolrich, M. W., Mackay, C. E., and Jezzard, P. (2010). Assessment of arterial arrival times derived from multiple inversion time pulsed arterial spin labeling MRI. Magn. Reson. Med. 63, 641-647. doi: 10.1002/mrm.22256

Majeed, W., Magnuson, M., Hasenkamp, W., Schwarb, H., Schumacher, E. H., Barsalou, L., et al. (2011). Spatiotemporal dynamics of low frequency BOLD fluctuations in rats and humans. Neuroimage 54, 1140-1150.

Majeed, W., Magnuson, M., and Keilholz, S. D. (2009). Spatiotemporal dynamics of low frequency fluctuations in BOLD fMRI of the rat. J. Magn. Reson. Imaging 30, 384-393.

Martin, C., Zheng, Y., Sibson, N. R., Mayhew, J. E., and Berwick, J. (2013). Complex spatiotemporal haemodynamic response following sensory stimulation in the awake rat. Neuroimage 66, 1-8. doi: 10.1016/j.neuroimage.2012.10.006

Martindale, J., Berwick, J., Martin, C., Kong, Y., Zheng, Y., and Mayhew, J. (2005). Long duration stimuli and nonlinearities in the neural-haemodynamic coupling. J. Cereb. Blood Flow Metab. 25, 651-661. doi: 10.1038/sj.jcbfm. 9600060

Matsui, T., Murakami, T., and Ohki, K. (2016). Transient neuronal coactivations embedded in globally propagating waves underlie resting-state functional connectivity. Proc. Natl. Acad. Sci. U.S.A. 113, 6556-6561. doi: 10.1073/pnas. 1521299113

Mitra, A., Snyder, A. Z., Blazey, T., and Raichle, M. E. (2015). Lag threads organize the brain's intrinsic activity. Proc. Natl. Acad. Sci. U.S.A. 112, E2235-E2244.

Mitra, A., Snyder, A. Z., Hacker, C. D., and Raichle, M. E. (2014). Lag structure in resting-state fMRI. J. Neurophysiol. 111, 2374-2391.

Moeller, S., Yacoub, E., Olman, C. A., Auerbach, E., Strupp, J., Harel, N., et al. (2010). Multiband multislice GE-EPI at 7 tesla, with 16-fold acceleration using partial parallel imaging with application to high spatial and temporal wholebrain fMRI. Magn. Reson. Med. 63, 1144-1153. doi: 10.1002/mrm.22361

Murphy, K., Birn, R. M., Handwerker, D. A., Jones, T. B., and Bandettini, P. A. (2009). The impact of global signal regression on resting state correlations: are anti-correlated networks introduced? Neuroimage 44, 893-905.

Nowak, L. G., and Bullier, J. (1997). "The timing of information transfer in the visual system," in Extrastriate Cortex in Primates, Vol. 12, eds K. S. Rockland, J. H. Kaas, and A. Peters (Boston, MA: Springer), 205-241.

Puckett, A. M., Mathis, J. R., and DeYoe, E. A. (2014). An investigation of positive and inverted hemodynamic response functions across multiple visual areas. Hum. Brain Mapp. 35, 5550-5564. doi: 10.1002/hbm.22569

Raut, R. V., Mitra, A., Marek, S., Ortega, M., Snyder, A. Z., Tanenbaum, A., et al. (2019). Organization of propagated intrinsic brain activity in individual humans. Cereb. Cortex 30, 1716-1734. doi: 10.1093/cercor/bhz198

Rayshubskiy, A., Wojtasiewicz, T. J., Mikell, C. B., Bouchard, M. B., Timerman, D., Youngerman, B. E., et al. (2014). Direct, intraoperative observation of $\sim 0.1$ $\mathrm{Hz}$ hemodynamic oscillations in awake human cortex: implications for fMRI. Neuroimage 87, 323-331. doi: 10.1016/j.neuroimage.2013.10.044

Roc, A. C., Wang, J., Ances, B. M., Liebeskind, D. S., Kasner, S. E., and Detre, J. A. (2006). Altered hemodynamics and regional cerebral blood flow in patients with hemodynamically significant stenoses. Stroke 37, 382-387.

Salimi-Khorshidi, G., Douaud, G., Beckmann, C. F., Glasser, M. F., Griffanti, L., Smith, S. M., et al. (2014). Automatic denoising of functional MRI data: combining independent component analysis and hierarchical fusion of classifiers. Neuroimage 90, 449-468. doi: 10.1016/j.neuroimage.2013.11.046

Sato, K., Sadamoto, T., Hirasawa, A., Oue, A., Subudhi, A. W., Miyazawa, T., et al. (2012). Differential blood flow responses to $\mathrm{CO}(2)$ in human internal and external carotid and vertebral arteries. J. Physiol. 590, 3277-3290. doi: 10.1113/jphysiol.2012.230425

Scholvinck, M. L., Maier, A., Ye, F. Q., Duyn, J. H., and Leopold, D. A. (2010). Neural basis of global resting-state fMRI activity. Proc. Natl. Acad. Sci. U.S.A. 107, 10238-10243.

Setsompop, K., Gagoski, B. A., Polimeni, J. R., Witzel, T., Wedeen, V. J., and Wald, L. L. (2012). Blipped-controlled aliasing in parallel imaging for simultaneous multislice echo planar imaging with reduced g-factor penalty. Magn. Reson. Med. 67, 1210-1224. doi: 10.1002/mrm.23097

Sette, G., Baron, J. C., Mazoyer, B., Levasseur, M., Pappata, S., and Crouzel, C. (1989). Local brain haemodynamics and oxygen metabolism in cerebrovascular 
disease. Positron emission tomography. Brain 112(Pt 4), 931-951. doi: 10.1093/ brain/112.4.931

Smith, S. M., Beckmann, C. F., Andersson, J., Auerbach, E. J., Bijsterbosch, J., Douaud, G., et al. (2013). Resting-state fMRI in the human connectome project. Neuroimage 80, 144-168. doi: 10.1016/j.neuroimage.2013.05.039

Smith, S. M., Miller, K. L., Moeller, S., Xu, J., Auerbach, E. J., Woolrich, M. W., et al. (2012). Temporally-independent functional modes of spontaneous brain activity. Proc. Natl. Acad. Sci. U.S.A. 109, 3131-3136.

Stroh, A., Adelsberger, H., Groh, A., Rühlmann, C., Fischer, S., Schierloh, A., et al. (2013). Making waves: initiation and propagation of corticothalamic Ca2+ waves in vivo. Neuron 77, 1136-1150.

Tak, S., Polimeni, J. R., Wang, D. J., Yan, L., and Chen, J. J. (2015). Associations of resting-state fMRI functional connectivity with flow-BOLD coupling and regional vasculature. Brain Connect. 5, 137-146. doi: 10.1089/brain.2014.0299

Tak, S., Wang, D. J., Polimeni, J. R., Yan, L., and Chen, J. J. (2014). Dynamic and static contributions of the cerebrovasculature to the resting-state BOLD signal. Neuroimage 84, 672-680. doi: 10.1016/j.neuroimage.2013.09.057

Takeda, Y., Hiroe, N., Yamashita, O., and Sato, M. A. (2016). Estimating repetitive spatiotemporal patterns from resting-state brain activity data. Neuroimage 133, 251-265. doi: 10.1016/j.neuroimage.2016.03.014

Taylor, A. J., Kim, J. H., and Ress, D. (2018). Characterization of the hemodynamic response function across the majority of human cerebral cortex. Neuroimage 173, 322-331. doi: 10.1016/j.neuroimage.2018.02.061

Tiecks, F. P., Lam, A. M., Aaslid, R., and Newell, D. W. (1995). Comparison of static and dynamic cerebral autoregulation measurements. Stroke 26, 1014-1019. doi: 10.1161/01.str.26.6.1014

Tong, Y., and Frederick, B. (2010). Time lag dependent multimodal processing of concurrent fMRI and near-infrared spectroscopy (NIRS) data suggests a global circulatory origin for low-frequency oscillation signals in human brain. Neuroimage 53, 553-564.

Tong, Y., and Frederick, B. (2012). Concurrent fNIRS and fMRI processing allows independent visualization of the propagation of pressure waves and bulk blood flow in the cerebral vasculature. Neuroimage 61, 1419-1427.

Tong, Y., and Frederick, B. (2014a). Studying the spatial distribution of physiological effects on BOLD signals using ultrafast fMRI. Front. Hum. Neurosci. 8:196. doi: 10.3389/fnhum.2014.00196

Tong, Y., and Frederick, B. (2014b). Tracking cerebral blood flow in BOLD fMRI using recursively generated regressors. Hum. Brain Mapp. 35, 5471-5485.

Tong, Y., and Hocke, L. M. (2014). Short repetition time multiband echo-planar imaging with simultaneous pulse recording allows dynamic imaging of the cardiac pulsation signal. Magn. Reson. Med. 72, 1268-1276.

Tong, Y., Hocke, L. M., and Frederick, B. B. (2019a). Low frequency systemic hemodynamic "noise" in resting state BOLD fMRI: characteristics, causes, implications, mitigation strategies, and applications. Front. Neurosci. 13:787. doi: $10.3389 /$ fnins.2019.00787

Tong, Y., Yao, J. F., Chen, J. J., and Frederick, B. D. (2019b). The resting-state fMRI arterial signal predicts differential blood transit time through the brain. J. Cereb. Blood Flow Metab. 39, 1148-1160. doi: 10.1177/0271678x17753329

Tong, Y., Hocke, L. M., Licata, S. C., and Frederick, B. (2012). Low-frequency oscillations measured in the periphery with near-infrared spectroscopy are strongly correlated with blood oxygen level-dependent functional magnetic resonance imaging signals. J. Biomed. Opt. 17:106004. doi: 10.1117/1.jbo.17.10. 106004

Tong, Y., Hocke, L. M., Nickerson, L. D., Licata, S. C., Lindsey, K. P., and Frederick, B. (2013). Evaluating the effects of systemic low frequency oscillations measured in the periphery on the independent component analysis results of resting state networks. Neuroimage 76, 202-215.
Tong, Y., Lindsey, K. P., Hocke, L. M., Vitaliano, G., Mintzopoulos, D., and Frederick, B. D. (2017). Perfusion information extracted from resting state functional magnetic resonance imaging. J. Cereb. Blood Flow Metab. 37, 564576. doi: $10.1177 / 0271678 \times 16631755$

Van Essen, D. C., Smith, S. M., Barch, D. M., Behrens, T. E., Yacoub, E., Ugurbil, K., et al. (2013). The WU-minn human connectome project: an overview. Neuroimage 80, 62-79. doi: 10.1016/j.neuroimage.2013.05.041

Vanni, M. P., Chan, A. W., Balbi, M., Silasi, G., and Murphy, T. H. (2017). Mesoscale mapping of mouse cortex reveals frequency-dependent cycling between distinct macroscale functional modules. J. Neurosci. 37, 7513-7533. doi: 10.1523/jneurosci.3560-16.2017

Vincent, J. L., Patel, G. H., Fox, M. D., Snyder, A. Z., Baker, J. T., Van Essen, D. C., et al. (2007). Intrinsic functional architecture in the anaesthetized monkey brain. Nature 447, 83-86.

Weber, B., Keller, A. L., Reichold, J., and Logothetis, N. K. (2008). The microvascular system of the striate and extrastriate visual cortex of the macaque. Cereb. Cortex 18, 2318-2330. doi: 10.1093/cercor/bhm259

Willie, C. K., Macleod, D. B., Shaw, A. D., Smith, K. J., Tzeng, Y. C., Eves, N. D., et al. (2012). Regional brain blood flow in man during acute changes in arterial blood gases. J. Physiol. 590, 3261-3275. doi: 10.1113/jphysiol.2012.228551

Wise, R. G., Ide, K., Poulin, M. J., and Tracey, I. (2004). Resting fluctuations in arterial carbon dioxide induce significant low frequency variations in BOLD signal. Neuroimage 21, 1652-1664. doi: 10.1016/j.neuroimage.2003. 11.025

Wodarz, R. (1980). Watershed infarctions and computed tomography. A topographical study in cases with stenosis or occlusion of the carotid artery. Neuroradiology 19, 245-248.

Xiong, B., Li, A., Lou, Y., Chen, S., Long, B., Peng, J., et al. (2017). Precise cerebral vascular atlas in stereotaxic coordinates of whole mouse brain. Front. Neuroanat. 11:128. doi: 10.3389/fnana.2017.00128

Yao, J. F., Wang, J. H., Yang, H. S., Liang, Z., Cohen-Gadol, A. A., Rayz, V. L., et al. (2019). Cerebral circulation time derived from fMRI signals in large blood vessels. J. Magn. Reson. Imaging 50, 1504-1513. doi: 10.1002/jmri. 26765

Zhang, D., Snyder, A. Z., Shimony, J. S., Fox, M. D., and Raichle, M. E. (2010). Noninvasive functional and structural connectivity mapping of the human thalamocortical system. Cereb. Cortex 20, 1187-1194.

Zhang, R., Zuckerman, J. H., Giller, C. A., and Levine, B. D. (1998). Transfer function analysis of dynamic cerebral autoregulation in humans. Am. J. Physiol. 274(1 Pt 2), H233-H241. doi: 10.1152/ajpheart.1998.274.1.h233

Zhang, R., Zuckerman, J. H., and Levine, B. D. (2000). Spontaneous fluctuations in cerebral blood flow: insights from extended-duration recordings in humans. Am. J. Physiol. Heart Circ. Physiol. 278, H1848-H1855. doi: 10.1152/ajpheart. 2000.278.6.H1848

Zülch, K.-J. (1985). The Cerebral Infarct: Pathology, Pathogenesis, and Computed Tomography. Berlin: Springer-Verlag.

Conflict of Interest: The authors declare that the research was conducted in the absence of any commercial or financial relationships that could be construed as a potential conflict of interest.

Copyright (c) 2020 Amemiya, Takao and Abe. This is an open-access article distributed under the terms of the Creative Commons Attribution License (CC BY). The use, distribution or reproduction in other forums is permitted, provided the original author(s) and the copyright owner(s) are credited and that the original publication in this journal is cited, in accordance with accepted academic practice. No use, distribution or reproduction is permitted which does not comply with these terms. 